\title{
Real-time monitoring of human guanine deaminase activity by an emissive guanine analog
}

Marcela S. Bucardo, You Wu, Paul T. Ludford, III, Yao Li, Andrea Fin, ${ }^{\dagger}$ Yitzhak Tor* $^{\star}$

Department of Chemistry and Biochemistry, University of California, San Diego, 9500 Gilman Drive, La Jolla, California, 92093, United States

${ }^{*}$ Corresponding Author: ytor@ucsd.edu

† Present Address: Dipartimento di Scienza e Tecnologia del Farmaco, University of Turin, Via P. Giuria, 9 , 10125 Turin, Italy

\section{Supporting Information}

1. Synthetic procedures $\quad$ S2

1.1. Scheme S1. Synthesis of thienopyrimidine-based analogs $\quad S 2$

1.2. Scheme S2. Synthesis of isothiazolepyrimidine precursor $\quad$ S2

1.3. Scheme S3. Synthesis of isothiazolopyrimidine-based analogs $\quad$ S2

1.4. Scheme S4. Synthesis of 4-aminoisothiazole-3-carboxamide S3

2. X-ray crystal structures $\quad S 4$

2.1. Experimental Summary $\quad S 4$

2.2. Table S1. Crystal data for th $\mathbf{G}_{N} \quad$ S5

2.3. Table S2. Crystal data for ${ }^{\text {th }} \mathbf{X}_{N} \quad S 6$

$\begin{array}{ll}\text { 2.4. Table S3. Crystal data for }{ }^{\mathrm{tz}} \mathbf{G}_{N} & \mathrm{~S} 7\end{array}$

2.5. Table S4. Crystal data for ${ }^{\text {tz }} \mathbf{X}_{N} \quad$ S8

3. Absorption and emission spectroscopy $\quad$ S9

3.1. Table S5. Complete photophysical properties of nucleobases $\quad$ S9

3.2. Table S6. $\mathrm{E}_{\mathrm{T}}(30)$ experimental values for water:dioxane mixtures $\quad \mathrm{S9}$

3.3. Figure S1. Nucleobase absorption and emission traces in water:dioxane mixtures $\quad \mathrm{S} 10$

3.4. Figure S2. Nucleobase absorption and emission traces in buffer solution at varying $\mathrm{pH} \quad \mathrm{S} 11$

4. Real-time monitoring of GDA reactions $\quad \mathrm{S} 12$

4.1. Figure S3. GDA-mediated deamination analysis by HPLC $\quad S 12$

4.2. Figure S4. Steady state absorption of guanine and analogs by GDA S13

5. MOE molecular docking results $\quad \mathrm{S} 14$

5.1. Figure S5. MOE docking of xanthine and analogs in GDA active site $\quad$ S14

5.2. Figure S6. MOE docking of guanine and analogs in GDA active site $\quad$ S15

6. Real-time monitoring of GDA and ${ }^{\text {tz }} \mathbf{G}_{N}$ reactions with inhibitors $\quad S 16$

6.1. Figure S7. Reaction monitoring of AICA, ATCA, and ICA as GDA inhibitors S16

7. Supplementary references $\quad S 16$ 
1. Synthetic procedures

The nucleobase analogs ${ }^{\mathrm{t} z} \mathbf{G}_{N},{ }^{\mathrm{t}} \mathbf{X}_{N},{ }^{\text {th }} \mathbf{G}_{N}$, and ${ }^{\text {th }} \mathbf{X}_{N}$ and the starting precursor methyl 4-aminoisothiazole3-carboxylate (1) were synthesized based on previously published procedures. ${ }^{1-4}$

1.1. Scheme $\mathbf{S} 1$. Synthesis of thienopyrimidine-based analogs, ${ }^{\text {th }} \mathbf{G}_{N}$ and ${ }^{\text {th }} \mathbf{X}_{\boldsymbol{N}}$.
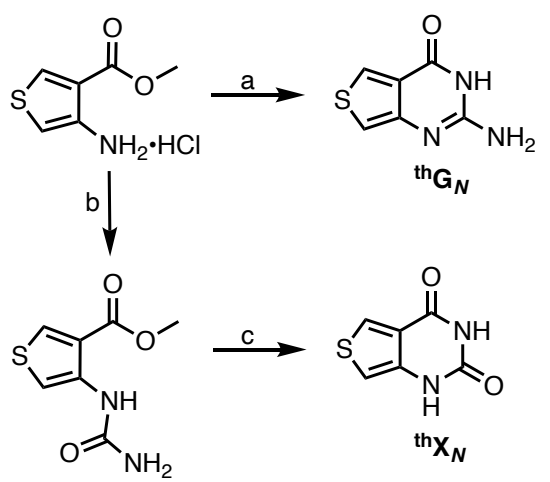

(a) Chloroformamidine hydrochloride, $\mathrm{DMSO}_{2}, 125^{\circ} \mathrm{C}, 2.5 \mathrm{~h}, 77 \%$. (b) $\mathrm{KOCN}$, Acetic acid, water, RT, overnight, $81 \%$. (c) $1 \mathrm{M} \mathrm{NaOMe}$ in $\mathrm{MeOH}, \mathrm{RT}$, overnight, $70 \%$.

1.2. Scheme S2. Synthesis of isothiazolepyrimidine precursor methyl 4-aminothiazole 3-carboxylate hydrochloride (1).

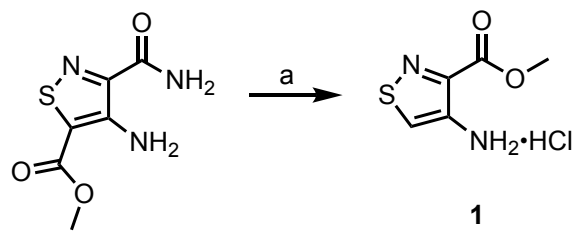

(a) i) $\mathrm{HCl}, 120^{\circ} \mathrm{C}, 1.5 \mathrm{~h}$. ii) $\mathrm{H}_{2} \mathrm{SO}_{4}, \mathrm{MeOH}, 65^{\circ} \mathrm{C}$, overnight, $66 \%$ over 2 steps.

1.3. Scheme S3. Synthesis of isothiazolo-pyrimidine-based analogs, ${ }^{\mathrm{tz}} \mathbf{G}_{\mathbf{N}}$ and ${ }^{\mathrm{tz}} \mathbf{X}_{\mathbf{N}}$, from precursor $\mathbf{4}$.

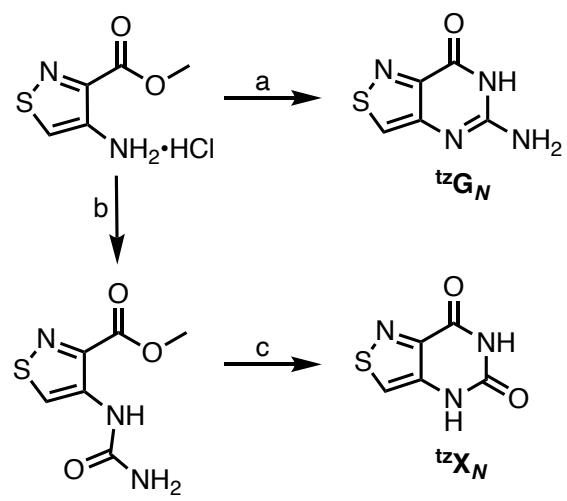

(a) Chloroformamidine hydrochloride, $\mathrm{DMSO}_{2}, 125^{\circ} \mathrm{C}, 2.5 \mathrm{~h}, 85 \%$. (b) $\mathrm{KOCN}$, Acetic acid, water, RT, overnight, $85 \%$. (c) $1 \mathrm{M} \mathrm{NaOMe}$ in $\mathrm{MeOH}, \mathrm{RT}$, overnight, $82 \%$. 
1.4. Scheme S4. Synthesis of 4-aminoisothiazole-3-carboxamide 2, (ATCA).
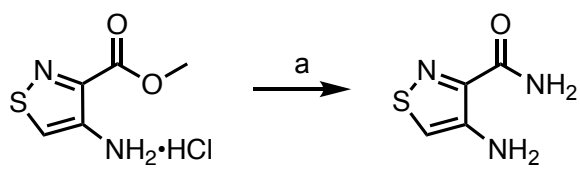

2

(a) $\mathrm{MeOH}, \mathrm{NH}_{3}, 60^{\circ} \mathrm{C}$, overnight, $87 \%$.

\section{4-aminoisothiazole 3-carboxamide (2)}

Solid $1(0.2 \mathrm{~g}, 0.1 \mathrm{mmol})$ is dissolved in cold anhydrous methanol $(7 \mathrm{~mL})$ in a $15 \mathrm{~mL}$ reaction vial with stir bar. Gaseous ammonia was bubbled through for 15 minutes and the vial was capped and left to stir overnight at $60{ }^{\circ} \mathrm{C}$. The reaction was then evaporated to dryness and subjected to purification by column chromatography $(0 \% \mathrm{MeOH}$ to $5 \% \mathrm{MeOH}$ in $\mathrm{DCM})$ to yield a yellow powder (0.13 g, 87\%). Rf: 0.33 (5\% methanol in DCM). ${ }^{1} \mathrm{H}$ NMR (500MHz, DMSO-d $)$ : $\delta 7.84$ (s, 1H), 7.61 (s, 1H), 7.46 (s, 1H), 5.73 (s 2H). ${ }^{13} \mathrm{C}$ NMR (125MHz, DMSO-d $): \delta 164.86,148.49,147.02,123.72$. ESI-HRMS calculated for $\left[\mathrm{C}_{4} \mathrm{H}_{6} \mathrm{~N}_{3} \mathrm{OS}\right]^{+} 144.0226$, found 144.0225 . 
2. X-ray crystal structures

\subsection{Experimental summary}

The single crystal $x$-ray diffraction studies were carried out on a Bruker Kappa APEX-II CCD diffractometer quipped with $\mathrm{Cu} \mathrm{K}_{\alpha}$ radiation $(\lambda=1.5478)$ or $\mathrm{Mo} \mathrm{K}_{\alpha}$ radiation $(\lambda=0.71073)$ at the UCSD Chemistry \& Biochemistry Small Molecule X-ray facility. Crystal blocks were mounted on a Cryoloop with Paratone oil. Data were collected in a nitrogen gas stream at $100(2) \mathrm{K}$ using $\phi$ and $\varpi$ scans. The data were integrated using the Bruker SAINT software program and scaled using SADABS software program. Solution by direct methods (SHELXT) produced a complete phasing model system consistent with the proposed structure. All nonhydrogen atoms were refined anisotropically by full-matrix least-squares (SHELLXL-2014). All carbon bonded hydrogens were placed using a rigid model. Their positions were constrained relative to their parent atom using the appropriate HFIX command SHELXL-2014. All other hydrogen atoms (H-bonding) were located in the difference map. Their relative positions were restrained using DFIX commands and their thermals freely refined.

Deposition numbers: 2073101, 2073102, 2073062, and 2073063. 


\subsection{Table S1. Crystal data and structure refinement for th $\mathbf{G}_{N}$}

Report date

Identification code

Empirical formula

Molecular formula

Formula weight

Temperature

Wavelength

Crystal system

Space group

Unit cell dimensions

Volume

Z

Density (calculated)

Absorption coefficient

$\mathrm{F}(000)$

Crystal size

Crystal color, habit

Theta range for data collection

Index ranges

Reflections collected

Independent reflections

Completeness to theta $=68.000^{\circ}$

Absorption correction

Max. and min. transmission

Refinement method

Data / restraints / parameters

Goodness-of-fit on $\mathrm{F}^{2}$

Final $\mathrm{R}$ indices $[\mathrm{I}>2 \operatorname{sigma}(\mathrm{I})]$

$\mathrm{R}$ indices (all data)

Extinction coefficient

Largest diff. peak and hole
2018-03-21

thG

C6 H5 N3 O S

C6 H5 N3 O S

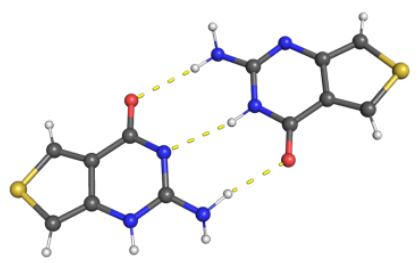

167.19

$100.0 \mathrm{~K}$

$1.54178 \AA$

Monoclinic

P $121 / \mathrm{c} 1$

$\mathrm{a}=7.2425(6) \AA$

$\alpha=90^{\circ}$.

$\mathrm{b}=14.8279(12) \AA$

$\beta=94.751(3)^{\circ}$.

$c=12.8685(11) \AA$

$\gamma=90^{\circ}$.
8

$1.613 \mathrm{Mg} / \mathrm{m}^{3}$

$3.680 \mathrm{~mm}^{-1}$

688

$0.231 \times 0.157 \times 0.084 \mathrm{~mm}^{3}$

Colorless Block

4.558 to $68.321^{\circ}$.

$-8<=\mathrm{h}<=8,-17<=\mathrm{k}<=17,-15<=\mathrm{l}<=14$

25913

$2519[\mathrm{R}(\mathrm{int})=0.0310, \mathrm{R}($ sigma $)=0.0171]$

$99.8 \%$

Semi-empirical from equivalents

0.3201 and 0.2149

Full-matrix least-squares on $\mathrm{F}^{2}$

$2519 / 6 / 223$

1.062

$\mathrm{R} 1=0.0301, \mathrm{wR} 2=0.0833$

$\mathrm{R} 1=0.0319, \mathrm{wR} 2=0.0849$

$\mathrm{n} / \mathrm{a}$

0.256 and -0.267 e. $\AA^{-3}$ 
2.3. Table S2. Crystal data and structure refinement for th $\mathbf{X}_{N}$

Report date

Identification code

Empirical formula

Molecular formula

Formula weight

Temperature

Wavelength

Crystal system

Space group

Unit cell dimensions

Volume

Z

Density (calculated)

Absorption coefficient

$\mathrm{F}(000)$

Crystal size

Crystal color, habit

Theta range for data collection

Index ranges

Reflections collected

Independent reflections

Completeness to theta $=25.000^{\circ}$

Absorption correction

Max. and min. transmission

Refinement method

Data / restraints / parameters

Goodness-of-fit on $\mathrm{F}^{2}$

Final $\mathrm{R}$ indices $[\mathrm{I}>2 \operatorname{sigma}(\mathrm{I})]$

$\mathrm{R}$ indices (all data)

Extinction coefficient

Largest diff. peak and hole
2018-04-04

THX01

C6 H4 N2 O2 S

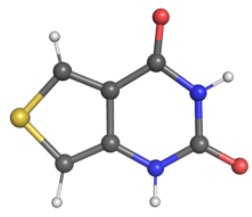

$\mathrm{C} 6 \mathrm{H} 4 \mathrm{~N} 2 \mathrm{O} 2 \mathrm{~S}$

168.17

$100.0 \mathrm{~K}$

$0.71073 \AA$

Monoclinic

P 1 21/c 1

$a=10.1968(16) \AA$

$\alpha=90^{\circ}$.

$\mathrm{b}=5.3149(8) \AA$

$\beta=102.119(4)^{\circ}$.

$\mathrm{c}=12.257(2) \AA$ $\gamma=90^{\circ}$.
4

$1.720 \mathrm{Mg} / \mathrm{m}^{3}$

$0.436 \mathrm{~mm}^{-1}$

344

$0.271 \times 0.117 \times 0.093 \mathrm{~mm}^{3}$

Colorless Block

2.043 to $28.278^{\circ}$.

$-13<=\mathrm{h}<=13,-6<=\mathrm{k}<=7,-16<=\mathrm{l}<=13$

8784

$1596[\mathrm{R}($ int $)=0.0582, \mathrm{R}($ sigma $)=0.0463]$

$100.0 \%$

Semi-empirical from equivalents

0.2482 and 0.2096

Full-matrix least-squares on $\mathrm{F}^{2}$

$1596 / 2$ / 108

1.075

$\mathrm{R} 1=0.0376, \mathrm{wR} 2=0.0963$

$\mathrm{R} 1=0.0435, \mathrm{wR} 2=0.1004$

$\mathrm{n} / \mathrm{a}$

0.361 and -0.331 e. $\AA^{-3}$ 
2.4. Table S3. Crystal data and structure refinement for ${ }^{\mathrm{tz}} \mathbf{G}_{N}$

Report date

2018-07-10

Identification code

tor119

Empirical formula

C5 H4 N4 O S

Molecular formula

C5 H4 N4 O S

Formula weight

168.18

Temperature

$100.0 \mathrm{~K}$

Wavelength

$0.71073 \AA$

Crystal system

Monoclinic

Space group

Unit cell dimensions

P $121 / \mathrm{c} 1$
$\mathrm{a}=3.6599(2) \AA$
$\alpha=90^{\circ}$.
$\mathrm{b}=11.2567(5) \AA$
$\beta=96.825(2)^{\circ}$.
$\mathrm{c}=15.0364(7) \AA$
$\gamma=90^{\circ}$.

Volume

615.09(5) $\AA^{3}$

Z

4

Density (calculated)

$1.816 \mathrm{Mg} / \mathrm{m}^{3}$

Absorption coefficient

$0.457 \mathrm{~mm}^{-1}$

$\mathrm{F}(000)$

344

Crystal size

$0.03 \times 0.005 \times 0.005 \mathrm{~mm}^{3}$

Crystal color, habit

light yellow needle

Theta range for data collection

2.266 to $26.720^{\circ}$.

Index ranges

$-4<=\mathrm{h}<=4,-13<=\mathrm{k}<=14,-18<=\mathrm{l}<=18$

Reflections collected

9691

Independent reflections

$1303[\mathrm{R}(\mathrm{int})=0.0555]$

Completeness to theta $=25.242^{\circ}$

$100.0 \%$

Absorption correction

Semi-empirical from equivalents

Max. and min. transmission

0.7455 and 0.6591

Refinement method

Full-matrix least-squares on $\mathrm{F}^{2}$

Data / restraints / parameters

$1303 / 0 / 100$

Goodness-of-fit on $\mathrm{F}^{2}$

1.046

Final $R$ indices $[\mathrm{I}>2 \operatorname{sigma}(\mathrm{I})]$

$\mathrm{R} 1=0.0337, \mathrm{wR} 2=0.0733$

$\mathrm{R}$ indices (all data)

$\mathrm{R} 1=0.0471, \mathrm{wR} 2=0.0784$

Largest diff. peak and hole

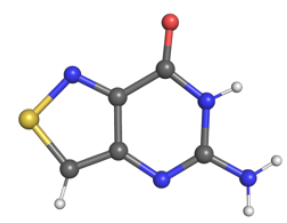




\subsection{Table S4. Crustal data and structure refinement for ${ }^{\mathrm{t}} \mathbf{X}_{N}$}

Report date

Identification code

Empirical formula

Molecular formula

Formula weight

Temperature

Wavelength

Crystal system

Space group

Unit cell dimensions

Volume

Z

Density (calculated)

Absorption coefficient

$\mathrm{F}(000)$

Crystal size

Crystal color, habit

Theta range for data collection

Index ranges

Reflections collected

Independent reflections

Completeness to theta $=25.242^{\circ}$

Absorption correction

Max. and min. transmission

Refinement method

Data / restraints / parameters

Goodness-of-fit on $\mathrm{F}^{2}$

Final $\mathrm{R}$ indices $[\mathrm{I}>2 \operatorname{sigma}(\mathrm{I})]$

$\mathrm{R}$ indices (all data)

Largest diff. peak and hole
2018-07-11

tor 120

$\mathrm{C} 5 \mathrm{H} 3 \mathrm{~N} 3 \mathrm{O} 2 \mathrm{~S}$

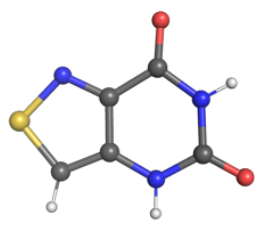

C5 H3 N3 O2 S

169.16

$100.0 \mathrm{~K}$

$0.71073 \AA$

Monoclinic

P $121 / \mathrm{c} 1$

$\mathrm{a}=6.9522(10) \AA$

$\alpha=90^{\circ}$.

$\mathrm{b}=12.2384(17) \AA$

$\beta=93.431(3)^{\circ}$.

$\mathrm{c}=6.9021(10) \AA$

$\gamma=90^{\circ}$.

$586.20(14) \AA^{3}$

4

$1.917 \mathrm{Mg} / \mathrm{m}^{3}$

$0.488 \mathrm{~mm}^{-1}$

344

$0.12 \times 0.08 \times 0.04 \mathrm{~mm}^{3}$

colorless block

2.935 to $26.736^{\circ}$.

$-8<=\mathrm{h}<=8,0<=\mathrm{k}<=15,0<=\mathrm{l}<=8$

1244

$1244[\mathrm{R}(\mathrm{int})=0.0362]$

$100.0 \%$

Semi-empirical from equivalents

0.491 and 0.402

Full-matrix least-squares on $\mathrm{F}^{2}$

1244 / 0 / 101

1.036

$\mathrm{R} 1=0.0362, \mathrm{wR} 2=0.0873$

$\mathrm{R} 1=0.0456, \mathrm{wR} 2=0.0927$

0.409 and -0.459 e. $\AA^{-3}$ 
3. Absorption and emission spectroscopy

3.1. Table S5. Complete photophysical properties of nucleobases

\begin{tabular}{|c|c|c|c|c|c|c|c|}
\hline & solvent & $\lambda_{\mathrm{abs}^{\mathrm{a}}}\left(\varepsilon^{\mathrm{b}}\right)$ & $\lambda_{\mathrm{em}}{ }^{\mathrm{a}}(\Phi)$ & $\Phi_{\varepsilon}$ & $\begin{array}{l}\text { Stokes } \\
\text { Shiftc }^{c}\end{array}$ & $\begin{array}{l}\text { Polarity } \\
\text { sensitivity }\end{array}$ & $\mathrm{p} K_{\mathrm{a}}(\mathrm{abs})$ \\
\hline \multirow[t]{2}{*}{${ }^{\mathrm{tz}} \mathbf{G}_{N}$} & water & $320(5.4 \pm 0.1)$ & $446(0.068 \pm 0.004)$ & 351 & 8.88 & 125.3 & $3.28,8.96$ \\
\hline & dioxane & $336(5.1 \pm 0.1)$ & $419(0.026 \pm 0.001)$ & 129 & 5.90 & & \\
\hline \multirow[t]{2}{*}{${ }^{\mathrm{t}} \mathrm{X}_{N}$} & water & $315(6.1 \pm 0.1)$ & $394(0.018 \pm 0.001)$ & 116 & 6.42 & 53.1 & 8.8 \\
\hline & dioxane & $315(6.4 \pm 0.01)$ & $375(0.001 \pm 0.001)$ & 11 & 5.08 & & \\
\hline \multirow[t]{2}{*}{${ }^{\text {th }} \mathbf{G}_{N}$} & water & $315(3.0 \pm 0.1)$ & $439(0.40 \pm 0.04)$ & 1179 & 8.97 & 137.4 & $4.41,10.19$ \\
\hline & dioxane & $325(3.0 \pm 0.1)$ & $423(0.40 \pm 0.05)$ & 1182 & 7.18 & & \\
\hline \multirow[t]{2}{*}{${ }^{\text {th }} \mathbf{X}_{N}$} & water & $308(3.2 \pm 0.1)$ & $420(0.46 \pm 0.05)$ & 1406 & 8.66 & 71.9 & 9.92 \\
\hline & dioxane & $303(3.6 \pm 0.1)$ & $383(0.065 \pm 0.002)$ & 242 & 6.95 & & \\
\hline
\end{tabular}

${ }^{a} \lambda_{\text {abs, max }}$ and $\lambda_{\text {em, max }}$ are in $\mathrm{nm} .{ }^{\mathrm{b}} \varepsilon$ is in $10^{3} \mathrm{M}^{-1} \mathrm{~cm}^{-1}$. ${ }^{\mathrm{c}}$ Stokes Shifts is in $10^{3} \mathrm{~cm}^{-1}$. Experiments done in triplicate.

3.2. Table S6. $\mathrm{E}_{\mathrm{T}}(30)$ experimental values for water:dioxane mixtures

\begin{tabular}{ccc} 
water:dioxane & $\begin{array}{c}\text { Reported } \mathrm{E}_{\mathrm{T}}(30) \\
\left(\mathrm{kcal} \mathrm{mol}{ }^{-1}\right)\end{array}$ & $\begin{array}{c}\text { Experimental } \mathrm{E}_{\mathrm{T}}(30) \\
\text { th } \mathrm{G}_{N} \text { and }{ }^{\mathrm{tz}} \mathrm{G}_{N},{ }^{\text {th }} \mathrm{X}_{N} \text { and }{ }^{\mathrm{tz}} \mathrm{X}_{N} \\
\left(\mathrm{kcal} \mathrm{mol}^{-1}\right)\end{array}$ \\
\hline $1: 0$ & 63.1 & - \\
$4: 1$ & 57.5 & $57.9,57.5$ \\
$3: 2$ & 55 & $54.8,54.9$ \\
$2: 3$ & 51.6 & $51.7,51.6$ \\
$1: 4$ & 48.3 & $47.7,48.1$ \\
$0: 1$ & 36.4 & $35.8,36.5$
\end{tabular}


3.3. Nucleobase absorption and emission traces in water:dioxane mixtures
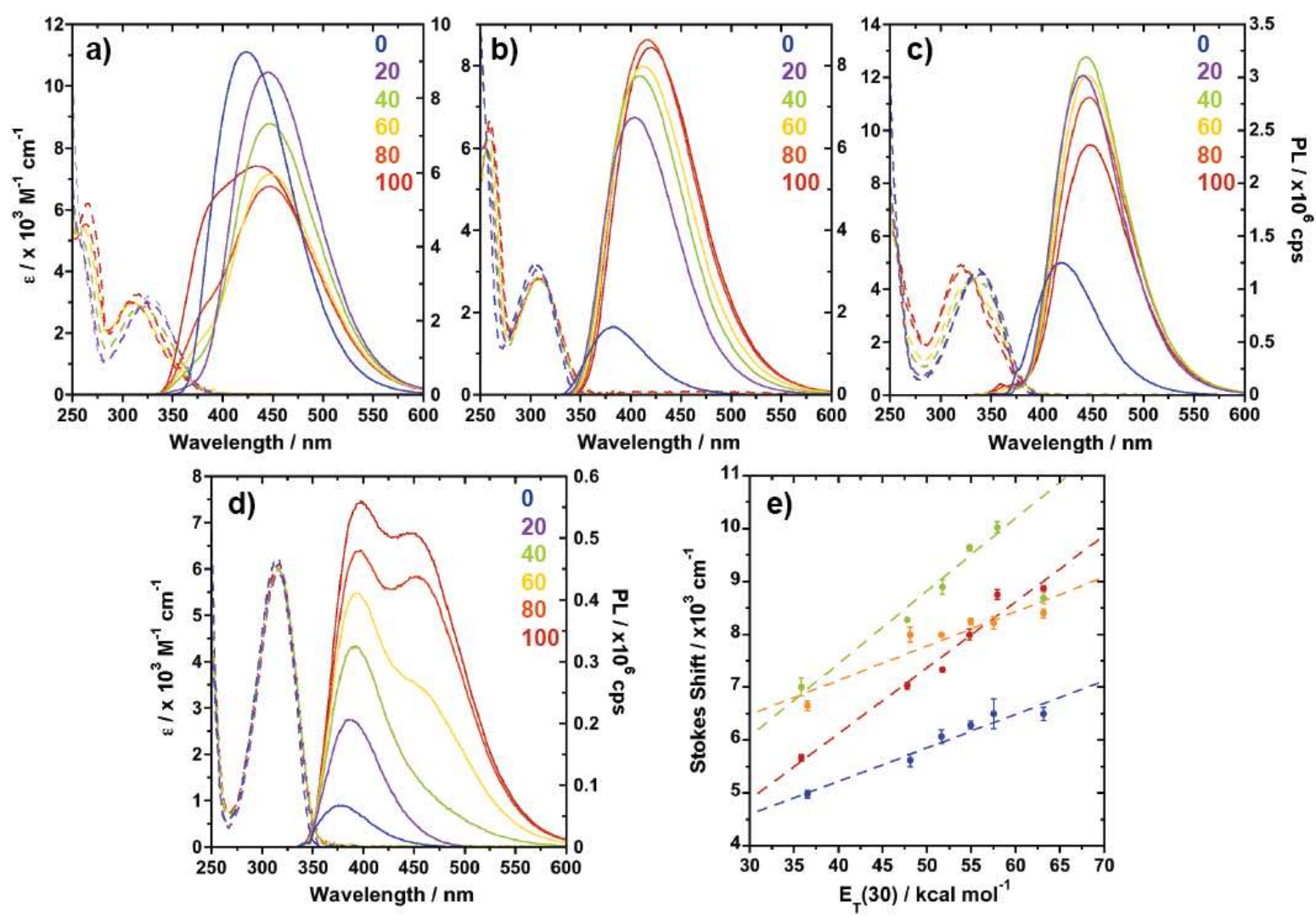

Figure S1. Absorption (dashed lines) and emission (solid lines) traces in water/dioxane mixtures from 0 to $100 \%$ water for (a) th $\mathbf{G}_{N}$, (b) ${ }^{\text {th }} \mathbf{X}_{N}$, (c) ${ }^{\text {tz }} \mathbf{G}_{N}$, and (d) ${ }^{\text {tz }} \mathbf{X}_{N}$. (e) Stokes shift correlation versus solvent polarity $\left[\mathrm{E}_{\mathrm{T}}(30)\right.$ of water/dioxane mixtures for ${ }^{\text {th }} \mathbf{G}_{N}$ (green), ${ }^{\text {th }} \mathbf{X}_{N}$ (red), ${ }^{\text {tz }} \mathbf{G}_{N}$ (blue), and ${ }^{\text {tz }} \mathbf{X}_{N}$ (orange)]. ). 
3.4. Nucleobase absorption and emission traces in buffer solution at varying $\mathrm{pH}$
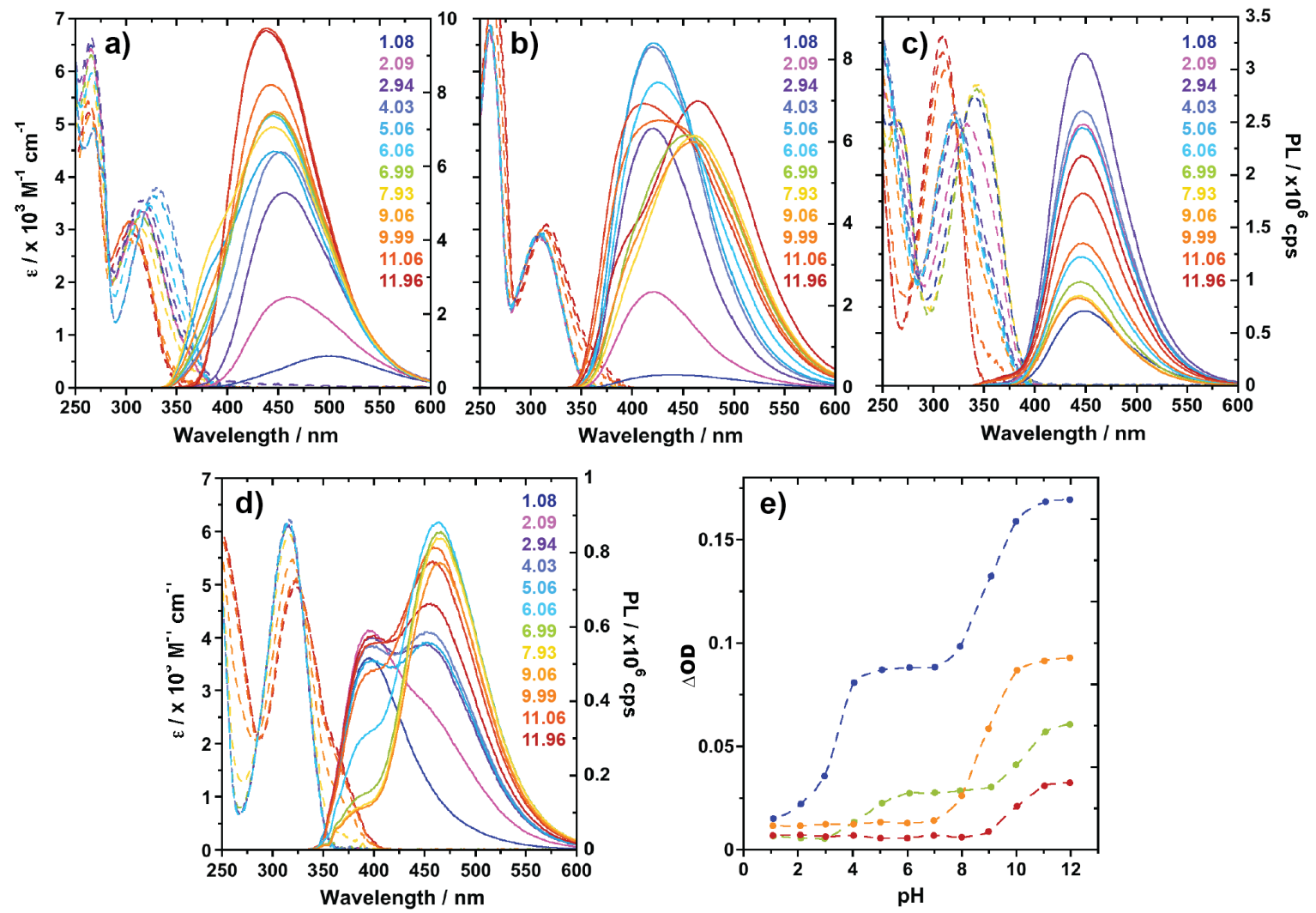

Figure S2. Absorption (dashed lines) and emission (solid lines) traces in buffer solution of varying $\mathrm{pH}$ for (a) ${ }^{\text {th }} \mathbf{G}_{N}$, (b) ${ }^{\text {th }} \mathbf{X}_{N},(c){ }^{\text {tz }} \mathbf{G}_{N}$, (d) ${ }^{\text {tz }} \mathbf{X}_{N}$. (e) Change in optical density versus $\mathrm{pH}$ for ${ }^{\text {th }} \mathbf{G}_{N}$ (green), ${ }^{\text {th }} \mathbf{X}_{N}$ (red), ${ }^{\text {tz }} \mathbf{G}_{N}$ (blue), and ${ }^{\text {tz }} \mathbf{X}_{N}$ (orange). 
4. Real-time monitoring of GDA reactions

4.1. GDA-mediated deamination analysis by HPLC
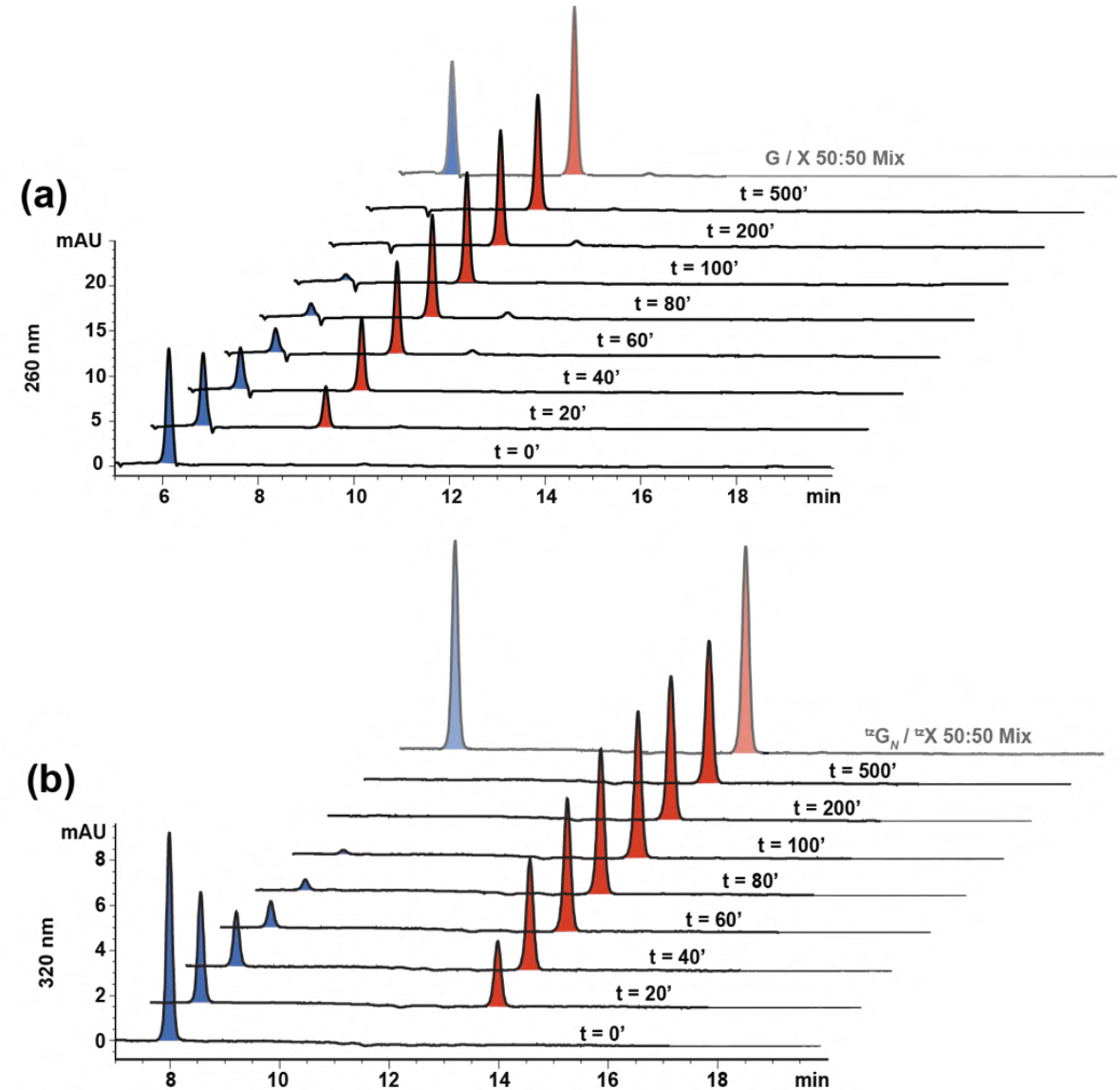

(c)

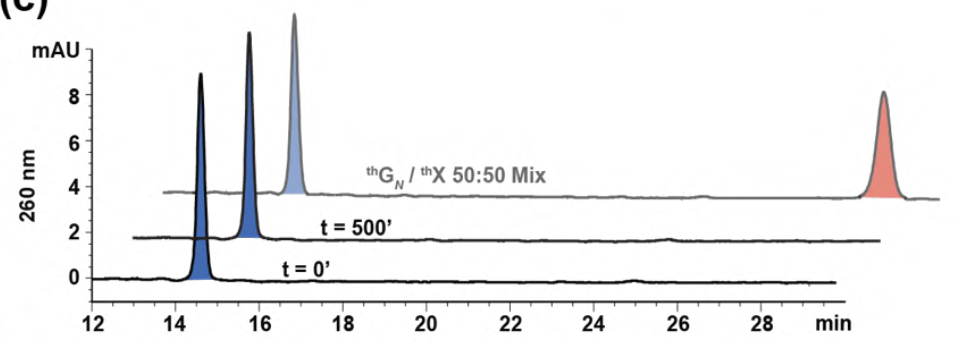

Figure S3. (a) Chromatogram shows enzymatic conversion of native guanine (blue) to xanthine (red) monitored at $260 \mathrm{~nm}$. (b) Enzymatic conversion of ${ }^{\mathrm{tz}} \mathbf{G}_{N}$ (blue) to ${ }^{\mathrm{tz}} \mathbf{X}_{N}$ (red) monitored at $320 \mathrm{~nm}$. (c) No enzymatic conversion of th $\mathbf{G}_{N}$ (blue) to ${ }^{\text {th }} \mathbf{X}_{N}$ (red) observed. Each data set shows a standard isomolar mixture of the starting material and product (transparent trace). 
4.2. Figure S4. Steady state absorption of guanine and analogs by GDA
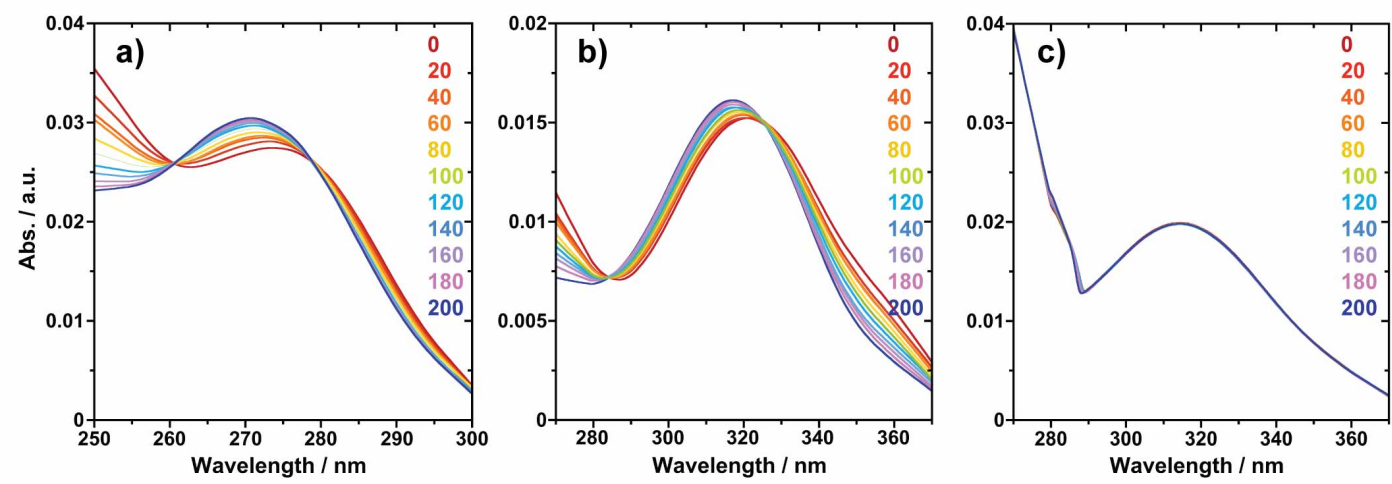

Figure S4. Steady state absorption traces of enzymatic conversion of (a) guanine to xanthine, (b) ${ }^{\text {tz }} \mathbf{G}_{N}$ to ${ }^{\text {tz }} \mathbf{X}_{N}$, and (c) ${ }^{\text {th }} \mathbf{G}_{N}$, over time from 0 (red) to 200 seconds (blue) with measurements made every 20 seconds. 
5. MOE docking results

5.1. Figure S5. MOE docking of xanthine and analogs in GDA active site
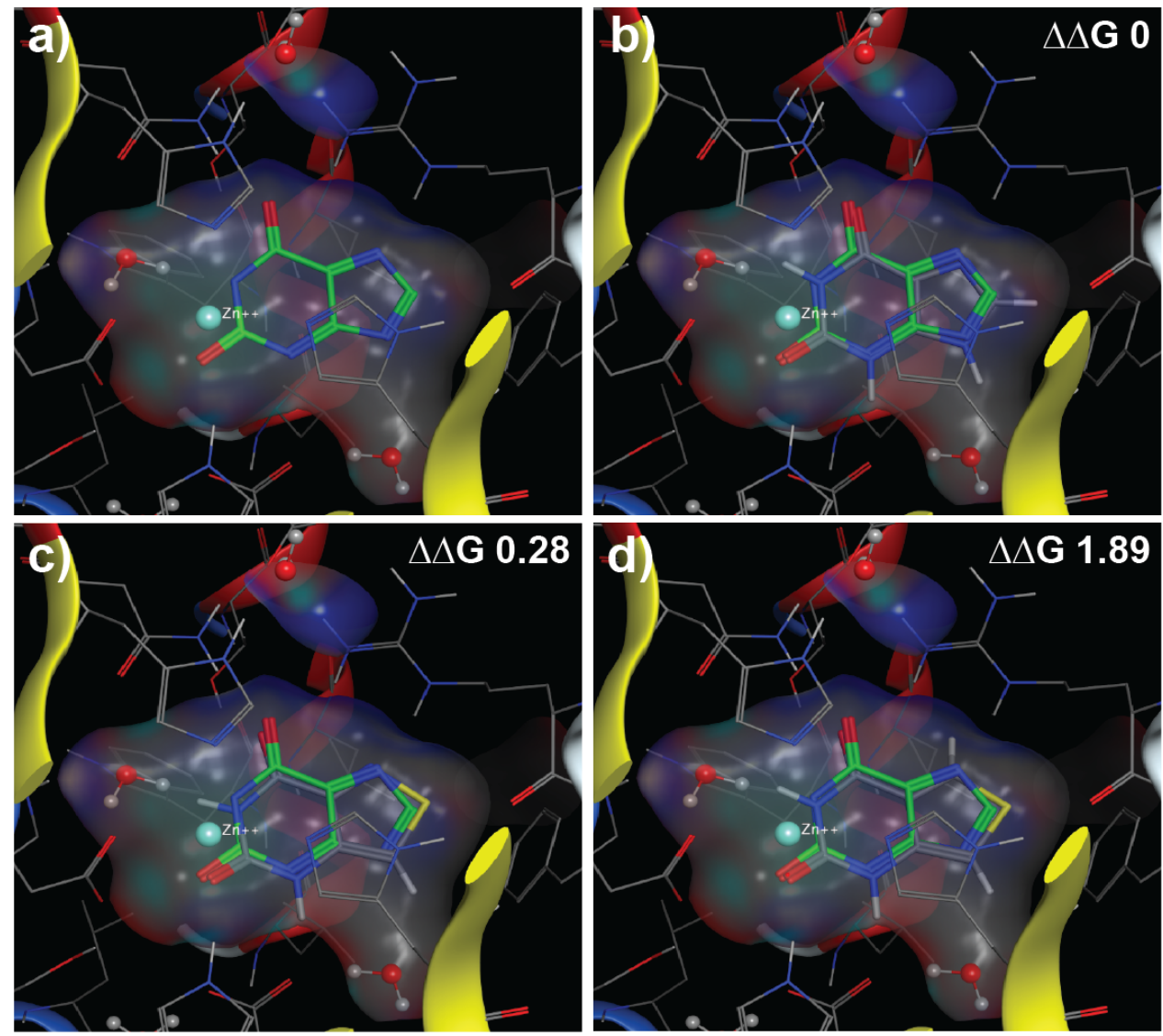

Figure S5. MOE docking images of xanthine and analogs in GDA active site (PDB 2UZ9) with calculated $\Delta \Delta G$ of docking (see methods) with xanthine as a reference. (a) Crystal structure active site with xanthine bound, (b) docking of native guanine, (c) docking of ${ }^{\mathrm{t}} \mathbf{X}_{N}$ with $\Delta \Delta \mathrm{G}$ of 0.28 , (d) and docking of th $\mathbf{X}_{N}$ with more positive $\Delta \Delta G$ of 1.89 when compared to ${ }^{\text {tz }} \mathbf{X}_{N}$. th $\mathbf{X}_{N}$ docking shows nucleobase analog structure deformation as well as steric hindrance at the position equivalent to the xanthine crystal substrate N7. The N7 moiety of ${ }^{\mathrm{t}} \mathbf{X}_{N}$ will likely hydrogen bond to Arg235, whereas the $\mathrm{CH}$ group of ${ }^{\text {th }} \mathbf{X}_{N}$ is sterically hindered in the active site. 
5. 2. Figure S6. MOE docking of guanine and analogs in GDA active site
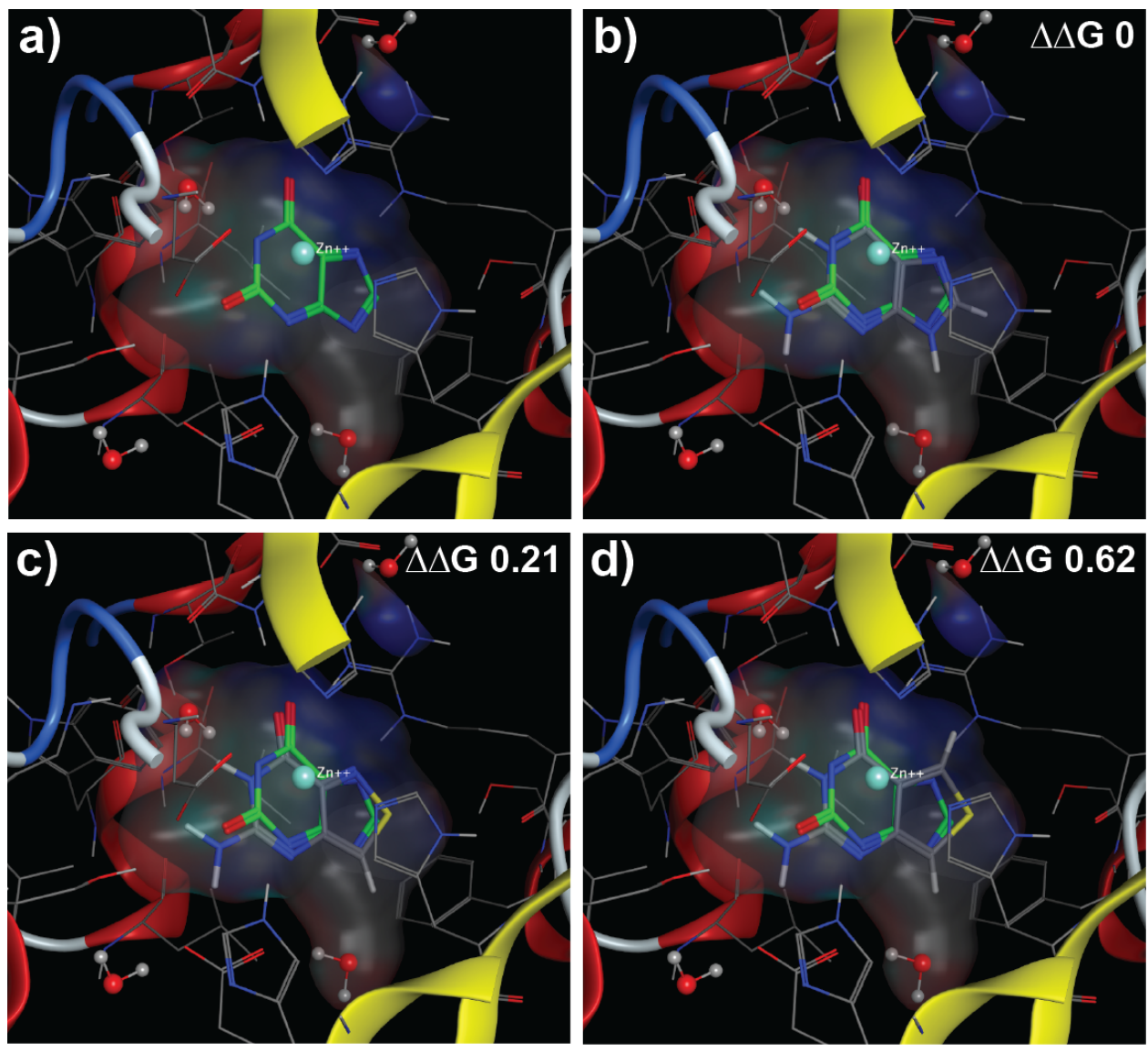

Figure S6. MOE docking images of guanine and analogs in GDA active site (PDB ID 2UZ9) with calculated $\Delta \Delta G$ (see methods) of docking with guanine as a reference. (a) Crystal structure active site with xanthine bound, (b) docking of native guanine, (c) docking of ${ }^{\text {tz }} \mathbf{G}_{N}$ with $\Delta \Delta G$ of 0.21 , and (d) docking of th $\mathbf{G}_{N}$ with more positive $\Delta \Delta \mathbf{G}$ of 0.62 when compared to ${ }^{\text {tz }} \mathbf{G}_{N} \cdot{ }^{\text {th }} \mathbf{G}_{N}$ docking shows nucleobase analog structure deformation as well as steric hindrance at the position equivalent to the xanthine crystal substrate N7, where the $\mathrm{CH}$ group of ${ }^{\text {th }} \mathbf{G}_{N}$ is sterically hindered by $\operatorname{Arg} 235$ in the active site. $\operatorname{Arg} 235$ likely provides a basic site that favors the $\mathrm{N} 7$ moiety in ${ }^{\mathrm{tz}} \mathbf{G}_{N}$ for substrate recognition. 
6. Real-time monitoring of GDA and ${ }^{\mathrm{tz}} \mathbf{G}_{N}$ reactions with inhibitors

6.1 Figure S7. Kinetics of AICA, ATCA, and ICA as GDA inhibitors
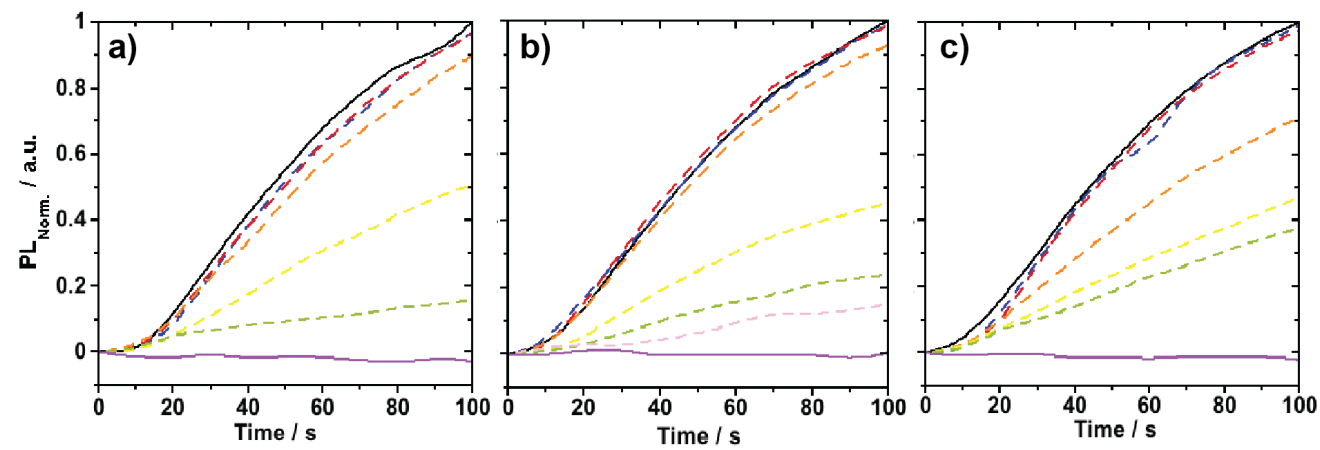

Figure S7. (a) Conversion of ${ }^{\mathrm{tz}} \mathbf{G}_{N}$ to ${ }^{\mathrm{tz}} \mathbf{X}_{N}$ by GDA in the presence of AICA at 0 (solid black), 0.1 (dashed blue), 1 (dashed red), 10 (dashed orange), 100 (dashed yellow), $1000 \mu \mathrm{M}$ (green dashed), and finally $1000 \mu \mathrm{M}$ without GDA (solid purple). (b) Conversion of ${ }^{\mathrm{tz}} \mathbf{G}_{N}$ to ${ }^{\mathrm{tz}} \mathbf{X}_{\boldsymbol{N}}$ by GDA in the presence of ATCA at 0 (solid black), 0.1 (dashed blue), 1 (dashed red), 10 (dashed orange), 100 (dashed yellow), 250 (dashed green), $500 \mu \mathrm{M}$ (dashed pink), and lastly $500 \mu \mathrm{M}$ without GDA (solid purple). (c) Conversion of ${ }^{\mathrm{tz}} \mathbf{G}_{N}$ to ${ }^{\mathrm{tz}} \mathbf{X}_{\boldsymbol{N}}$ by GDA in the presence of ICA at 0 (solid black), 10 (dashed blue), 100 (dashed red), 1000 (dashed orange), 2000 (dashed yellow), $3000 \mu \mathrm{M}$ (dashed green), and lastly $3000 \mu \mathrm{M}$ without GDA.

7. Supplementary references

1) Shin, D.; Sinkeldam, R. W.; Tor, Y. Emissive RNA Alphabet. J. Am. Chem. Soc. 2011, 133 (38), 14912-14915.

2) Rovira, A. R.; Fin, A.; Tor, Y. Chemical Mutagenesis of an Emissive RNA Alphabet. J. Am. Chem. Soc. 2015, 137 (46), 14602-14605.

3) Srivatsan, S. G.; Weizman, H.; Tor, Y. A Highly Fluorescent Nucleoside Analog Based on Thieno[3,4-d]Pyrimidine Senses Mismatched Pairing. Org. Biomol. Chem. 2008, 6 (8), 13341338.

4) Rovira, A. R.; Fin, A.; Tor, Y. Expanding a Fluorescent RNA Alphabet: Synthesis, Photophysics and Utility of Isothiazole-Derived Purine Nucleoside Surrogates. Chem. Sci. 2017, 8 (4), 2983-2993. 